\title{
Fungal infective endocarditis of polytetrafluoroethylene pulmonary valve with Paecilomyces species following tetralogy of Fallot correction
}

\author{
Sumeet Anand*, Swaminathan Vaidyanathan, Mustafa Janeel, Neville A. G. Solomon
}

Department of Pediatric Cardiac Surgery, Apollo Children's Hospital, Chennai, Tamil Nadu, India

Received: 31 January 2020

Accepted: 17 March 2020

\section{*Correspondence:}

Dr. Sumeet Anand,

E-mail: sumeet_a@yahoo.in

Copyright: ( ) the author(s), publisher and licensee Medip Academy. This is an open-access article distributed under the terms of the Creative Commons Attribution Non-Commercial License, which permits unrestricted non-commercial use, distribution, and reproduction in any medium, provided the original work is properly cited.

\begin{abstract}
Fungal infective endocarditis of the prosthetic pulmonary valve in non immunocompromised host is growing phenomena attributed to the increased use of prosthetic materials. High mortality and neurovascular sequalae is commonly seen in such case if treatment is delayed. Often misdiagnosed as bacterial endocarditis due to closely resembling clinical features and lack of inexpensive and readily available laboratory tests, the diagnosis is often delayed. High index of suspicion and early surgical intervention is needed for early diagnosis and management.
\end{abstract}

Keywords: Fungal endocarditis, Pulmonary thromboembolism, PTFE, Pacilomycetes, Tetralogy of fallot

\section{INTRODUCTION}

Fungal infective endocarditis of prosthetic valve is a grave and rare complication post cardiac surgery. The diagnosis of fungal endocarditis is often delayed given the low index of suspicion. The associated morbidity and mortality associated with fungal endocarditis is very high $(40-60 \%)$ and often requires surgical intervention. Recurrence of fungal endocarditis is well documented hence regular follow up is required. ${ }^{1}$

Right sided prosthetic valve endocarditis is usually seen in patients with immune compromised status, or those having undergone catheter based procedure or in IV drug abusers. $^{2-5}$ However it can also be seen in immunocompetent host and a great majority of it represent infection acquired in the healthcare setup.

Right sided vegetation are more common followed by left sided and bilateral vegetations; right side having a better prognosis compared to the left sided endocarditis. ${ }^{6}$ Fungal endocarditis is also associated with increased morbidity with complications such as embolization, septic shock, multi organ failure, disseminated fungal infection, stroke, progressive heart failure and acute renal failure ultimately leading to mortality.

The clinical features of fungal endocarditis resemble those of bacterial endocarditis. Prolonged fever, weight loss, weakness, fatigue predominate the clinical picture.

We present here the case of a 15 year old girl who was diagnosed to have culture negative bacterial endocarditis on presentation. However the tissue sent for HPE revealed the growth of fungi after which the antimicrobial treatment was modified.

\section{CASE REPORT}

A 15-year-old girl underwent intracardiac repair for tetrology of fallot 5 years ago with a polytetrafluoroethylene (PTFE) bicuspid valve replacement of pulmonary valve. She remained asymptomatic post-surgery and was on regular cardiac medications. She began having fever intermittently for the last 6 months, with weight loss and dyspnoea on exertion for the past few weeks. She was investigated and diagnosed as prosthetic valve endocarditis and treated 
with 2 months of broad spectrum antibiotics at her native place with little improvement in symptoms. Echo done at her native place had revealed $11 \times 8 \mathrm{~mm}$ size vegetation over the pulmonary valve. Initial blood cultures sent at our institution had revealed the growth of staphylococcus hominis but it was presumed to be a contaminant and she was started on a regimen of cefuroxime and vancomycin as a standard regime of treatment for bacterial endocarditis. Fever continued to persist and she complained of increasing breathlessness. A repeat echo done revealed an increase in size of the vegetation to $14 \times 14$ with RVOT gradient of $74 \mathrm{mmHg}$. It was decided to take her up for urgent surgery. Her dyspnoea got worse overnight and a CT pulmonary angiogram was done which revealed filling defect in the right and left pulmonary artery suggestive of thromboembolism. Intraoperatively the PTFE prosthetic pulmonary valve was found to be covered with vegetation, with vegetation fragments extending into the MPA and LPA.

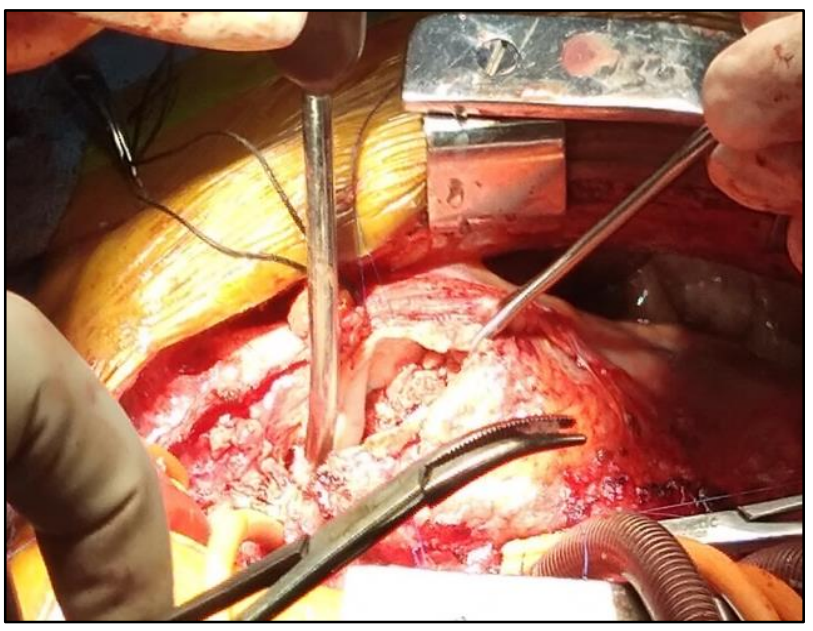

Figure 1: Extension of pulmonary vegetation into the pulmonary artery.

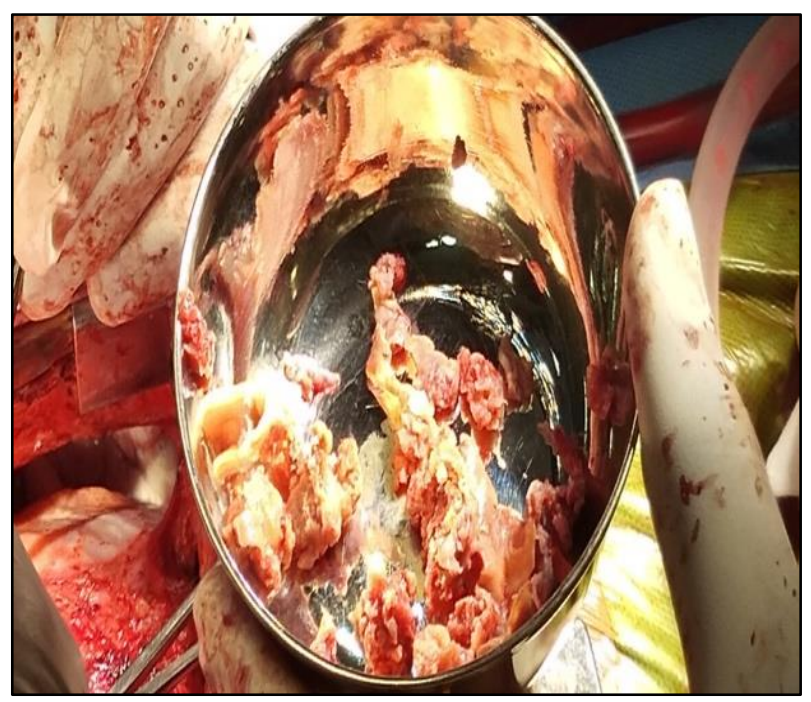

Figure 2: The extracted vegetation from the valve and pulmonary artery.
The pulmonary valve was excised completely and a bioprosthetic valve was put in the pulmonary position. Postoperatively she had an uneventful course and was discharged on the 11th postoperative day. HPE of the tissue revealed presence of hyphal elements. Antimicrobial treatment was accordingly changed and she was started on IV amphotericin and oral voriconazole. The organism was isolated to be pacilomycetes species sensitive to both the anti-fungal drugs.

\section{DISCUSSION}

Fungal infections are difficult to treat and even more difficult to diagnose. Clinically fungal endocarditis don't differ much in presentation from bacterial endocarditis with rise in temperature and malaise being the predominant symptoms. Diagnostic dilemma also comes into picture when a fungal infective endocarditis is misdiagnosed as a bacterial one and a course of antibiotic is started for a period of $4-6$ weeks (as per guidelines) instead of early surgery as indicated in fungal endocarditis. Incidence of fungal endocarditis has been steadily on the rise, more so in the younger population with prosthetic valves, recipients of broad spectrum antibiotics, immunocompromised patients and those with indwelling catheters. A review article published in 2017 about fungal endocarditis in paediatrics found that there have been 192 reported cases of fungal endocarditis. The overall mortality from fungal endocarditis in pediatric patients was reported to be $56.2 \% .^{7-9}$ The mortality rates were found to be significantly lower in the surgically treated group compared to the medically treated group ( $8 \%$ vs $70 \%$ respectively). Another study published in the American Journal of Cardiology in 2013 reported that out of the 516 suspected infective endocarditis patients, $7 \%$ were found to have fungal endocarditis of which $81 \%$ were diagnosed using the blood culture and $19 \%$ using the surgical/ histopathology findings. ${ }^{7}$

In-vitro growth of fungi in culture media can take upto weeks which often delays the diagnosis and treatment and is often a determining factor for mortality. ${ }^{8}$ Detecting blood stream fungal infection can be done via direct cultures or via serological/molecular tests such as beta D glucan, galactomannan, mannan antigen and PCR. ${ }^{8}$ Using blood cultures for diagnosing fungal endocarditis is not reliable. Serological/molecular tests also yield false positive results many a times, and confirmation is often possible only by histopathological examination of infected tissue or Polymerase chain reaction.

Candida is the most commonly implicated fungi followed by filamentous fungi and histoplasma.,8 Candida Albicans accounts for almost $50 \%$ of all fungal endocarditis. Filamentous fungi are more commonly seen in patients having h/o IV drug abuse and in patients with prosthetic valve endocarditis. Large mobile vegetations, vegetations involving prosthetic valve, persistent sepsis despite continuing antibiotic therapy for 5-7 days are all indications for early surgery. Once the diagnosis is 
established, surgery should be carried out at the earliest to prevent embolization of vegetation which is more often seen in the first few days of starting the antimicrobial therapy. ${ }^{3}$ Recurrence rate in fungal endocarditis have been reported to be between $8-18 \%$.

The genus pacilomycetes was 1st described in 1907 by Bainier as a saprophytic mould. ${ }^{2}$ Pacilomycetes are worldwide in occurrence and are isolated from soil and decaying matter. They are a common contaminant or sterile solution as they are resistant to most sterilisation techniques. ${ }^{5}$ The species is rarely pathogenic for humans and is implicated as causative organism for keratitis, endophthalmitis, onychomycosis, pneumonitis, endocarditis and sinusitis in immunocompetent hosts. In immunosuppressive hosts the pathogen mainly manifests as a disseminated disease. P. lilacus and $P$. Variotti are reported as the most pathogenic strains. ${ }^{5}$ Infective endocarditis caused by Paecilomyces is becoming increasingly common. Most cases of Paecilomyces have been reported as cutaneous infections in patients receiving solid organ transplantation students or lymphomas. The source for endocarditis is usually another site of infection or a prior intervention/ cardiac surgery. Paecilomyces is commonly found in solutions used for sterilising medical equipments since it is resistant to antimicrobial action of most commercially available disinfectant and sterilising solutions. ${ }^{5}$ The incubation period is long and treatment with antibiotics for bacterial endocarditis in most cases encourages the growth and multiplication of the fungi. ${ }^{2,8}$ The organism develops as large vegetations over the prosthetic material and can eventually embolize like it did in our case leading to acute pulmonary thromboembolism. Surgical removal of the prosthesis (a class I indication) and identification and susceptibility testing of the pathogen are the two most important principles in treatment of patients with Paecilomyces infection. ${ }^{5,8}$

\section{CONCLUSION}

The diagnosis of fungal endocarditis in our case was a post-operative diagnosis. Although blood cultures grew staphylococcus hominis, specific tests such as PCR and BDG were not carried out. High index of suspicion is the key to diagnosis of fungal endocarditis. Clinical diagnosis should be supplemented with the use of specific tests such as BDG, galactomannan, mannan antigen and PCR in patients suspected of endocarditis has h/o prosthetic valves implantation and histopathological examination of the specimen in culture negative cases. Recurrence with fungal endocarditis is common as these are indolent species. Periodic follow up of postoperative patient, adequate treatment with appropriate anti-fungals and early diagnosis is the key in prolonging the survival of such patients.
Funding: No funding sources

Conflict of interest: None declared

Ethical approval: Not required

\section{REFERENCES}

1. Kalish SB, Goldschmidt R, Li C, Knop R, Cook FV, Wilner $\mathrm{G}$, et al. Infective endocarditis caused by paecilomyces varioti. Am J Clin Pathol. 1982;78(2):249-52.

2. Haldane EV, MacDonald JL, Gittens WO, Yuce K, Rooyen CE. Prosthetic valvular endocarditis due to the fungus paecilomyces. Canadian Med Asso J. 1974;111(9):963.

3. Pettersson GB, Coselli JS, Hussain ST, Griffin B, Blackstone EH, Gordon SM, et al The American Association for Thoracic Surgery (AATS) consensus guidelines: surgical treatment of infective endocarditis: executive summary. J Thorac Cardiovasc Surg. 2017;6(153):1241-58.

4. Arora H, Mohan R, Agrawal D, Wankhade P, Sachdeva S, Nindra P. Recurrent prosthetic pulmonary valve endocarditis in repaired tetralogy of fallot. J Asso Phys India. 2018;66(12):86-8.

5. Schinabeck MK, Ghannoum MA. Human hyalohyphomycoses: a review of human infections due to acremonium spp, paecilomyces spp, penicillium spp, and scopulariopsis spp. J Chemotherap. 2003;15(2):5-15.

6. Siciliano RF, Gualandro DM, Sejas ON, Ignoto BG, Caramelli B, Mansur AJ, et al. Outcomes in patients with fungal endocarditis: a multicenter observational cohort study. Int $\mathbf{J}$ Infect Dis. 2018;77:48-52.

7. Sun XL, Zhang J, Wang GG, Zhuang XF, Yang YM, Zhu J, et al. Comparison of characteristics and short-term outcome from fungal infective endocarditis in prosthetic valve endocarditis versus native valve endocarditis. Am J Cardiol. 2013;112(1):111-6.

8. Pasha AK, Lee JZ, Low SW, Desai H, Lee KS, Mohajer M. Fungal endocarditis: update on diagnosis and management. Am J Med. 2016;129(10):1037-43.

9. Ganesan V, Ponnusamy SS, Sundaramurthy R. Fungal endocarditis in paediatrics: a review of 192 cases (1971-2016). Cardiol Young. 2017;27(8):1481-7.

Cite this article as: Anand S, Vaidyanathan S, Janeel M, Solomon NAG. Fungal infective endocarditis of polytetrafluoroethylene pulmonary valve with Paecilomyces species following tetralogy of Fallot correction. Int Surg J 2020;7:1666-8. 